\title{
Electron heating by large-amplitude shear Alfvén waves in the upper chromosphere with a force-free magnetic configuration
}

\author{
J. I. Sakai and S. Saito
}

\begin{abstract}
Laboratory for Plasma Astrophysics, Faculty of Engineering, Toyama University, 3190 Gofuku, Toyama 930-8555, Japan
e-mail: sakaijun@eng.u-toyama.ac.jp
\end{abstract}

Received 26 September 2005 / Accepted 18 January 2006

\begin{abstract}
Aims. We investigate how large-amplitude shear Alfvén waves (AWs) can heat the upper chromosphere with a magnetic force-free configuration.

Methods. We used a 2D3V fully relativistic electromagnetic particle-in-cell simulation.

Results. We found that the shear AWs with linear polarization and about one period can excite quasi-electrostatic waves driven by the modified two-stream instability, resulting in electron heating within a short time $t \simeq 50 \omega_{\mathrm{ci}}^{-1}$. The results could be applied to the electron heating in the upper chromosphere where small-scale magnetic flux tubes are interacting with each other, making shear magnetic field structures between them.
\end{abstract}

Key words. acceleration of particles - Sun: chromosphere - waves

\section{Introduction}

Observations with NASA's Transition Region and Coronal Explorer (TRACE) spacecraft have revealed the location of an unidentified coronal heating energy source (Ashwanden et al. 2000), showing that the main corona heating occurs at a height not greater than about $16000 \mathrm{~km}$ above the photosphere. The new observations contradict the conventional theory (for reviews see, e.g., Narain \& Ulmschneider 1990, 1996), which relies on heating the loops uniformly.

Furusawa \& Sakai (2000) presented simulation results of the collision of two flux tubes in the quiet photospheric network of the solar photosphere. They found that shock waves appear during the collision of two magnetic flux tubes, when two magnetic flux tubes with weak electric current collide with each other. The shock waves so generated can subsequently collide with another flux tube. Sakai et al. (2000) investigated the interaction process of the shock with the flux tube. They found that, during the collision of a shock wave with a magnetic flux tube with weak electric current, surface Alfvén waves (AWs) can be generated and can then propagate along the flux tube. However, when the shock wave collides with a magnetic flux tube with strong current, body AWs can then be generated and also propagate along the flux tube.

The problem for coronal heating is to understand the mechanisms that convert the kinetic energy of the convective motions near the photosphere to heat in the corona, as well as heating processes in the chromosphere. The many theoretical models of coronal heating can be divided into two categories: wave heating and DC heating. Among the wave-heating models, Sakai et al. (2001, and references therein) presented a new model of chromospheric loop heating based on their MHD simulation results. There, surface AWs and upward plasma-flows from the footpoints of the small-scale magnetic flux tubes can be effectively dissipated through magnetic reconnection near the chromospheric current sheets that may exist near the interaction regions of several magnetic flux tubes. The energy-dissipation process of the AWs in the above paper was treated within the MHD approximation.

Recently Tsiklauri et al. (2005a,b) showed the importance of collisionless damping processes of the shear AWs in heating plasmas by using a $2 \mathrm{D} 3 \mathrm{~V}$, fully relativistic electromagnetic particle-in-cell (PIC) simulation. They performed PIC simulations of shear AW interaction with density inhomogeneity in collisionless plasma that is one-dimensional across the uniform magnetic field. They found that due to the differences in local Alfvén speed, progressive distortion (phase mixing) of the AW front generates electrostatic fields that are nearly parallel to the magnetic field, which accelerates electrons via Landau damping. They also found that the shear AW amplitude decay law in the kinetic regime is the same as in the MHD approximation described by Heyvaerts \& Priest (1983).

Sakai et al. (2005) investigated the nonlinear dynamics of large amplitude shear AW in collisionless plasmas by using PIC simulation. They found that when the amplitude, $A=\delta B / B_{0}$, of the shear AW is larger than $A_{\mathrm{c}}=\frac{v_{\mathrm{th}, \mathrm{i}}}{c} \frac{\omega_{\mathrm{pe}}}{k c} \frac{\omega_{\mathrm{pe}}}{\omega_{\mathrm{ce}}}$, the shear AW spontaneously becomes unstable for the modified two-stream instability (MTSI), resulting in the excitation of small-scale quasielectrostatic waves with electric fields parallel to the uniform magnetic field. They found that the electrons are heated mostly in a direction parallel to the magnetic field due to the quasielectrostatic waves excited by the MTSI. Subsequently, the shear AW becomes unstable with strong transverse modulation, resulting in the excitation of ion acoustic waves that can heat ions. About $70 \%$ of the AW energy can be converted to plasma heating of both electrons and ions. The plasma heating time is within about $50 \omega_{\mathrm{ci}}{ }^{-1}$, which is shorter than the collision time between protons and neutral hydrogens in the upper chromosphere. They 
also showed that the results could be very important for plasma heating of coronal loops in the upper chromosphere where the Alfvén velocity $v_{\mathrm{A}}$ is larger than the electron thermal velocity $v_{\text {th,e }}$.

In this paper, we investigate how large-amplitude shear AWs can heat chromospheric plasma with magnetic force-free configuration by using a $2 \mathrm{D} 3 \mathrm{~V}$ fully relativistic electromagnetic PIC simulation. We found that the shear AWs with linear polarization and about one period can excite quasi-electrostatic waves driven by the MTSI, resulting in electron heating within a short time $t \simeq 50 \omega_{\mathrm{ci}}{ }^{-1}$. The results could be applied to the electron heating in the upper chromosphere where small-scale magnetic flux tubes are interacting each other to cause shear magnetic field structures between them. In Sect. 2 we present our simulation model. In Sect. 3 we present our simulation results, while in the final section we summarize our results.

\section{Simulation model}

We consider the upper chromosphere where small-scale flux tubes may emerge from and return to the photosphere with a typical height of about $2000 \mathrm{~km}$ within the chromosphere, shown in Fig. 1 of the paper by Sakai et al. (2001). We assume for simplicity that the interaction region where the two small-scale magnetic flux tubes contact each other has shear magnetic field structure of force-free magnetic configuration. We use $2 \mathrm{D} 3 \mathrm{~V}$, fully relativistic electromagnetic particle-in-cell (PIC) code, modified from 3D3V TRISTAN code (Buneman 1993) to investigate how the shear AW propagating from a lower chromosphere damps in the region of force-free magnetic configuration. The system size is $L_{x}=3300, L_{y}=800 \Delta$, where $\Delta(=1)$ is the grid size. The model force-free magnetic configuration is given by

$B_{x}(y)=B_{0} \sin \frac{y}{L}$,
$B_{z}(y)=B_{0} \cos \frac{y}{L}$,

where $L=L_{y} / 2 \pi \Delta$. The periodic boundary conditions for both $x$ and $y$ directions are imposed on the particles and fields. The ratio of ion mass to the electron mass is $m_{\mathrm{i}} / m_{\mathrm{e}}=16$, and the simulation time step is $\omega_{\mathrm{pe}} \Delta t=0.05$. We take the ratio of ion mass to the electron mass to be 16 , instead of 1836 , mainly because if we use a mass ratio of 1836 , then we need a big simulation system to keep the dynamics of ion Larmor motion, as well as a long simulation run, to follow the whole dynamics associated with damping of the shear AW. These simulations are impossible for the existing computer power. The Alfvén velocity is $v_{\mathrm{A}}=0.125 \mathrm{c}$. The ratio of electron cyclotron frequency to the plasma frequency is $\omega_{\mathrm{ce}} / \omega_{\mathrm{pe}}=0.5$, the Debye length is $v_{\text {th,e }} / \omega_{\text {pe }}=1 \Delta$, plasma beta is $\beta=0.08$, and skin depth is $c / \omega_{\text {pe }}=10 \Delta$. The electron thermal velocity is $v_{\text {th,e }}=0.1 c$, electron Larmor radius is $\rho_{\mathrm{e}}=v_{\mathrm{th}, \mathrm{e}} / \omega_{\mathrm{ce}}=2 \Delta$, the ion thermal velocity is $v_{\mathrm{th}, \mathrm{i}}=0.025 c$, and ion Larmor radius is $\rho_{\mathrm{i}}=v_{\mathrm{th}, \mathrm{i}} / \omega_{\mathrm{ci}}=8 \Delta$. This choice of model parameters is determined as follows: first we choose the plasma beta as $\beta=0.08$ and the electron thermal velocity as $v_{\text {th,e }}=0.1 c$, because we take the Debye length as $1 \Delta$ and the simulation time step is taken as $\omega_{\mathrm{pe}} \Delta t=0.05$, Then the ratio of electron cyclotron frequency to the plasma frequency is determined as $\omega_{\text {ce }} / \omega_{\text {pe }}=0.5$.

To excite the shear AW with linear polarization near the lefthand boundary $(x=5)$ in the simulation domain, we impose a current of the following form:

$$
\frac{\partial E_{z}}{\partial t}=-\delta B \sin \left(\omega_{\mathrm{d}} t\right)\left(0.5\left(\tanh \left(\frac{\left(t-t_{1}\right)}{t_{0}}\right)-\tanh \left(\frac{\left(t-t_{2}\right)}{t_{0}}\right)\right)\right)
$$

where $\omega_{\mathrm{d}}$ is the driving AW frequency that was fixed at $\omega_{\mathrm{d}}=$ $0.5 \omega_{\text {ci }}$, which ensures that no significant ion-cyclotron damping is present. The amplitude $\delta B$ is taken as $\delta B=0.5 B_{0}$. The onset time $t_{1}$ and off-set time $t_{2}$ of the excitation are taken as $t_{1}=3.1 \omega_{\mathrm{ci}}{ }^{-1}$ and $t_{2}=14 \omega_{\mathrm{ci}}{ }^{-1}$ with the characteristic relaxation time $t_{0}=0.78 \omega_{\mathrm{ci}}{ }^{-1}$. By the above excitation period we will see that the linearly polarized shear AW with about one wavelength can be generated. The total energy in the system during the simulation time was conserved within about $1.1 \%$.

\section{Simulation results}

First we present the time evolution of physical quantities to understand the overall phenomena observed in the present simulation. Figure 1a shows the time evolution of the magnetic field energy in the system: $\iint \frac{B_{y}{ }^{2}}{2 \mu_{0}} \mathrm{~d} x \mathrm{~d} y$, and Fig. 1b shows the time evolution of the electric field energies in the system: $\iint \frac{E_{x}{ }^{2}}{2 \epsilon_{0}} \mathrm{~d} x \mathrm{~d} y$ (solid line), $\iint \frac{E_{y}{ }^{2}}{2 \epsilon_{0}} \mathrm{~d} x \mathrm{~d} y$ (dotted line), $\iint \frac{E_{z}{ }^{2}}{2 \epsilon_{0}} \mathrm{~d} x \mathrm{~d} y$ (dashed line). As seen in Fig. 1a, the magnetic field energy of $B_{y}$ associated with the shear AW begins to increase at about the onset time $t_{1}=3.1 \omega_{\mathrm{ci}}{ }^{-1}$ and its energy supply is decreased around the offset time $t_{2}=14 \omega_{\mathrm{ci}}{ }^{-1}$. The electric field energy of $E_{z}$ shown by the dashed line in Fig. 1b shows the same time evolution with $B_{y}$ energy. This means that the electric field $E_{z}$ can be excited self-consistently associated with the shear AW. From the maximum values of energies of both magnetic field and electric field associated with the shear AW in Figs. 1a and b, we can estimate the ratio $\frac{\delta E_{z}}{\delta B_{y}}=0.13$, which agrees with the theoretical estimate $\frac{\delta E_{z}}{\delta B_{y}}=\frac{v_{\mathrm{A}}}{c}=0.125$.

As seen in Fig. 1b the two components of the electric field energies $E_{x}$ and $E_{y}$ increase slowly. We investigate why these electric fields that should not exist associated with the shear AW can be generated. The reason why the electric field energy $E_{y}$ increases is that the excited shear AW propagates with elliptic polarization. As seen later, these electric fields play an important role in electron heating. Figure 1c shows the time evolution of electron kinetic energies: $K_{\mathrm{e} x}=\Sigma \frac{m_{\mathrm{e}} V_{x}^{2}}{2}$ (solid line), $K_{\mathrm{e} y}=\Sigma \frac{m_{\mathrm{e}} V_{y}{ }^{2}}{2}$ (dotted line), $K_{\mathrm{e} z}=\Sigma \frac{m_{\mathrm{e}} V_{z}^{2}}{2}$ (dashed line). Figure 1d shows the time evolution of ion kinetic energies: $K_{\mathrm{i} x}=\Sigma \frac{m_{\mathrm{i}} V_{x}{ }^{2}}{2}$ (solid line), $K_{\mathrm{i} y}=\Sigma \frac{m_{\mathrm{i}} V_{y}{ }^{2}}{2}$ (dotted line), $K_{\mathrm{i} z}=\Sigma \frac{m_{\mathrm{i}} V_{z}^{2}}{2}$ (dashed line). As seen in Fig. 1c the electron kinetic energies increase slowly coinciding with the electric field energies of $E_{x}$ and $E_{y}$. A small ion kinetic energy increase is also seen in Fig. 1d. The maximum shear AW energy at about $11 \omega_{\mathrm{ci}}{ }^{-1}$ is estimated from the summation of $\iint \frac{B_{y}{ }^{2}}{2 \mu_{0}} \mathrm{~d} x \mathrm{~d} y(\sim 2200)$ and $K_{\mathrm{i} y}=\Sigma \frac{m_{\mathrm{i}} V_{y}{ }^{2}}{2}(\sim 3400)$ thereby deducing the initial thermal energy, which gives us about 5600. On the other hand, the electron kinetic energy increases by about 3500 , which means that about $62 \%$ of the AW energy is converted to the electron heating through the quasi-electrostatic waves that are excited by the MTSI.

Next in Fig. 2 we show the spatial distribution of the generated magnetic field, $B_{y}$ associated with shear Alfvén waves at (a) $\omega_{\text {ci }} t=13.28$; (b) 29.68 ; and (c) 46.87 . Due to the periodic boundary condition in the $\mathrm{x}$-direction, the shear AW propagating in the negative $x$-direction appears from the right-side boundary as seen in Fig. 2a. As the magnetic field $B_{x}$ is null at $y=0,400$, and 800 , the shear AW cannot propagate around these points. Therefore the wave front of the shear AW is deformed as seen in Fig. 2. This deformation gives rise to the phase-mixing as discussed recently by Tsikulauri et al. (2005), but as seen in 
(a)

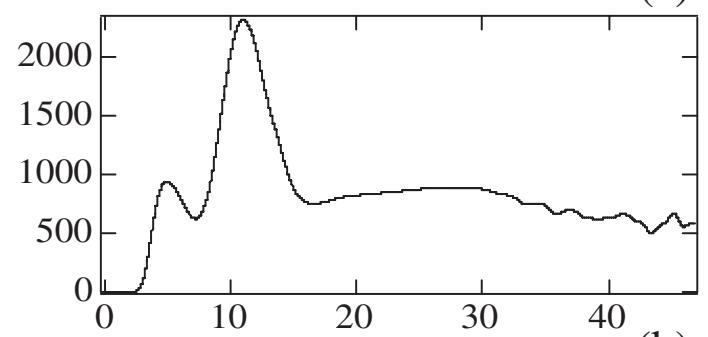

(b)

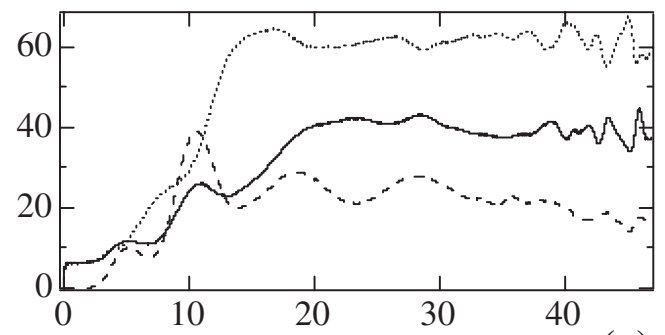

(c)

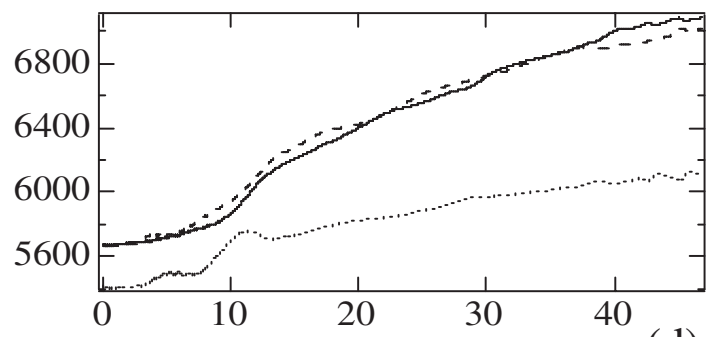

(d)

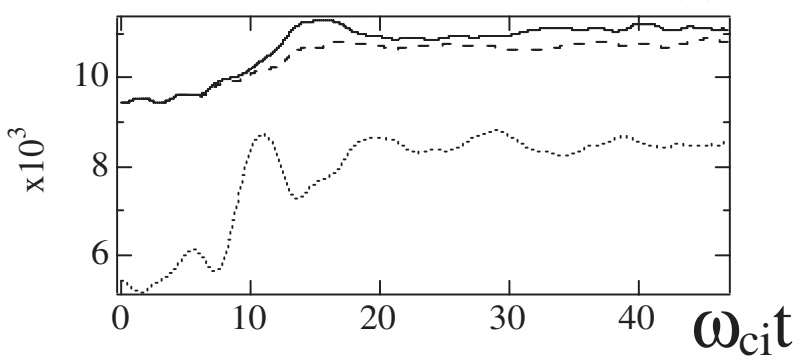

Fig. 1. Time evolution of a) magnetic field energy in the system; $\iint \frac{B_{y}{ }^{2}}{2 \mu_{0}} \mathrm{~d} x \mathrm{~d} y$, b) electric field energies in the system; $\iint \frac{E_{x}{ }^{2}}{2 \epsilon_{0}} \mathrm{~d} x \mathrm{~d} y$ (solid line), $\iint \frac{E_{y}{ }^{2}}{2 \epsilon_{0}} \mathrm{~d} x \mathrm{~d} y$ (dotted line), $\iint \frac{E_{z}{ }^{2}}{2 \epsilon_{0}} \mathrm{~d} x \mathrm{~d} y$ (dashed line), c) electron kinetic energies; $K_{\mathrm{e} x}=\Sigma \frac{m_{\mathrm{e}} V_{x}^{2}}{2}$ (solid line), $K_{\mathrm{e} y}=\Sigma \frac{m_{\mathrm{e}} V_{y}^{2}}{2}$ (dotted line), $K_{\mathrm{e} z}=\Sigma \frac{m_{\mathrm{e}} V_{z}^{2}}{2}$ (dashed line), d) ion kinetic energies; $K_{\mathrm{i} x}=\Sigma \frac{m_{\mathrm{i}} V_{x}^{2}}{2}$ (solid line), $K_{\mathrm{i} y}=\Sigma \frac{m_{\mathrm{i}} V_{y}{ }^{2}}{2}$ (dotted line), $K_{\mathrm{i} z}=\Sigma \frac{m_{\mathrm{i}} V_{z}^{2}}{2}$ (dashed line).

Fig. 3, which shows the time evolution of electric field $E_{x}$ at (a) $\omega_{\text {ci }} t=13.28$; (b) 29.68; and (c) 46.87, and that the generated electric field associated with the phase-mixing is weak. We find more strong excitation of electric fields $E_{x}$ with small-scale structures as seen in Fig. 3b. We also observed the small-scale structure of electric field $E_{y}$.

To understand the origin of the electric field $E_{x}$, we performed a space-time Fourier analysis of the electric field $E_{x}$ at $y=400 \Delta$, where the space data is taken in $600 \Delta<x<2648 \Delta$ and the time data is taken in $20 \omega_{\mathrm{ci}}{ }^{-1}<t<45.8 \omega_{\mathrm{ci}}{ }^{-1}$. Then we obtained the dispersion relation of $E_{x}$ that is shown in Fig. 4. To understand the origin of the observed quasi-electrostatic waves, we must consider the ion drift velocity $U_{\perp}$ to excite the shear
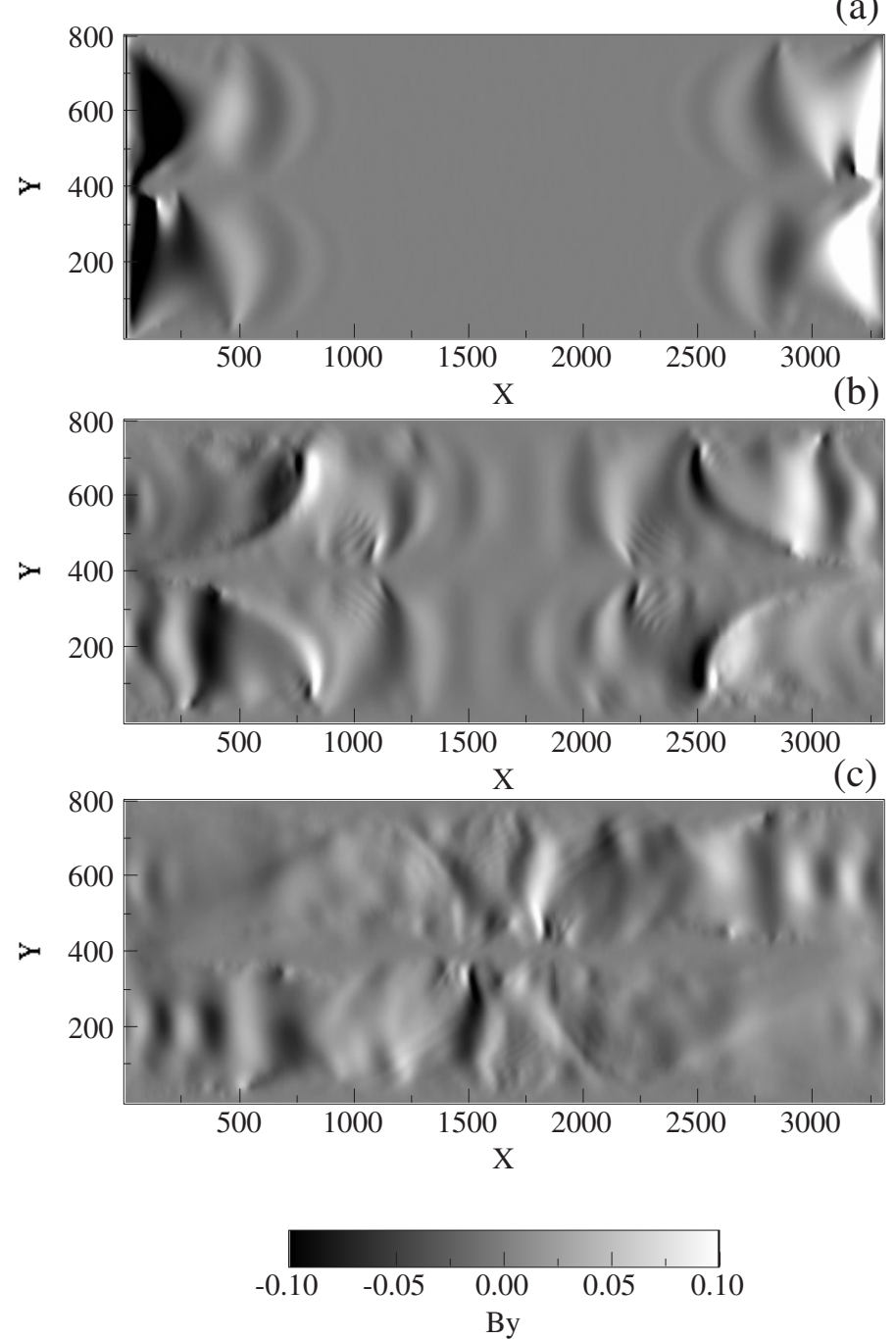

Fig. 2. The spatial distribution of the generated magnetic field, $B_{y}$ associated with shear Alfvén waves at a) $\omega_{\mathrm{ci}} t=13.28$, b) 29.68 , and c) 46.87 .

AW that is approximately given by $U_{\perp} / c=A \frac{k_{x} c}{\omega_{\mathrm{pe}}} \frac{\omega_{\mathrm{ce}}}{\omega_{\mathrm{pe}}}$, where $A=\delta B / B_{0}$ is the amplitude of the shear AW. If the shear AW has a larger amplitude than the critical amplitude $A_{\mathrm{c}}=\frac{v_{\mathrm{th}, \mathrm{i}}}{c} \frac{\omega_{\mathrm{pe}}}{k c} \frac{\omega_{\mathrm{pe}}}{\omega_{\mathrm{ce}}}$, the ion drift velocity $U_{\perp}$ to excite the shear AW becomes larger than the ion thermal velocity $v_{\text {th,i }}$. This condition is satisfied for the present simulation with $A=0.5, \omega_{\mathrm{ce}}=0.5 \omega_{\mathrm{pe}}$ and $\frac{k_{x} c}{\omega_{\mathrm{pe}}}=0.179$. The ion drift velocity $U_{\perp}$ is larger than the ion thermal velocity $v_{\text {th,i }}=0.025 c$. This situation is known to be unstable for the MTSI. The characteristics of the MTSI is summarized as follows. The unstable mode associated with the MTSI has $\operatorname{Re}[\omega] \sim \operatorname{Im}[\omega] \sim \omega_{\mathrm{pi}}\left(1+\omega_{\mathrm{pe}}{ }^{2} / \omega_{\mathrm{ce}}{ }^{2}\right)^{-1 / 2}$ for $k_{x} / k \sim\left(m_{\mathrm{e}} / m_{\mathrm{i}}\right)^{1 / 2}$, $\omega_{\text {ci }}<\operatorname{Re}[\omega]<\omega_{\text {ce }}$ and $\operatorname{Re}[\omega]>k_{x} v_{\text {th,e }}$. The phase velocity along the magnetic field associated with the MTSI is calculated from Fig. 4 and is about $0.14 c$, which is larger than the electron thermal velocity $v_{\text {th, }}=0.1 c$. As seen in Fig. 4 , the excited waves have a frequency in the range $\omega_{\text {ci }}<\operatorname{Re}[\omega]<\omega_{\text {ce }}$. Therefore we conclude that the quasi-electrostatic waves are generated by the MTSI caused by the ion transverse drift associated with the shear AW.

Figure 5 shows the electron velocity distribution functions of three components at $\omega_{\mathrm{ci}} t=0$ (dotted line) and 46.87 (solid line). As discussed before, the electron heating in the $y$ - and $z$-direction 
(a)
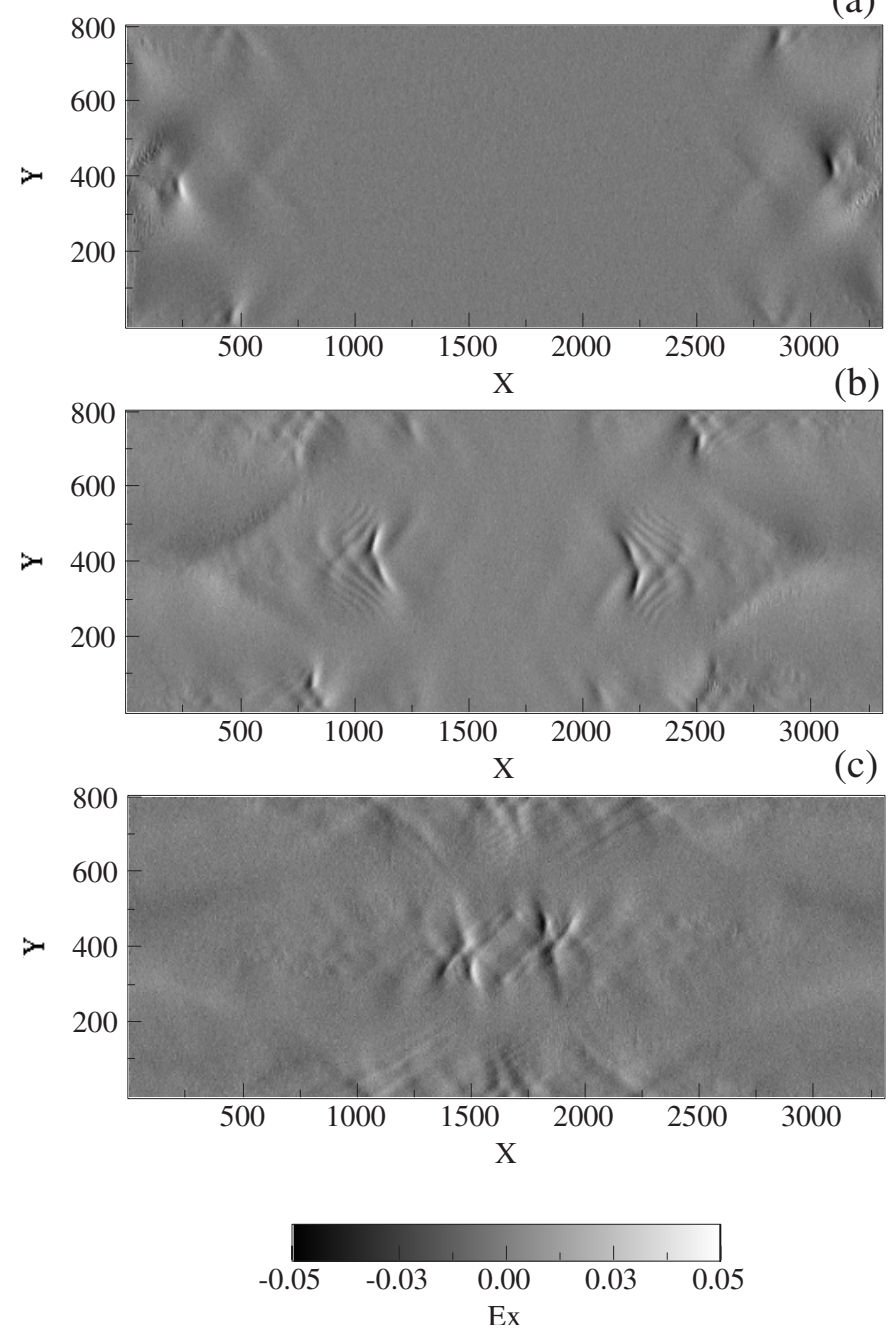

Fig. 3. The spatial distribution of the electric field, $E_{x}$ driven by modified two-stream instability associated with shear Alfvén waves at a) $\left.\omega_{\mathrm{ci}} t=13.28, \mathbf{b}\right) 29.68$, and c) 46.87.
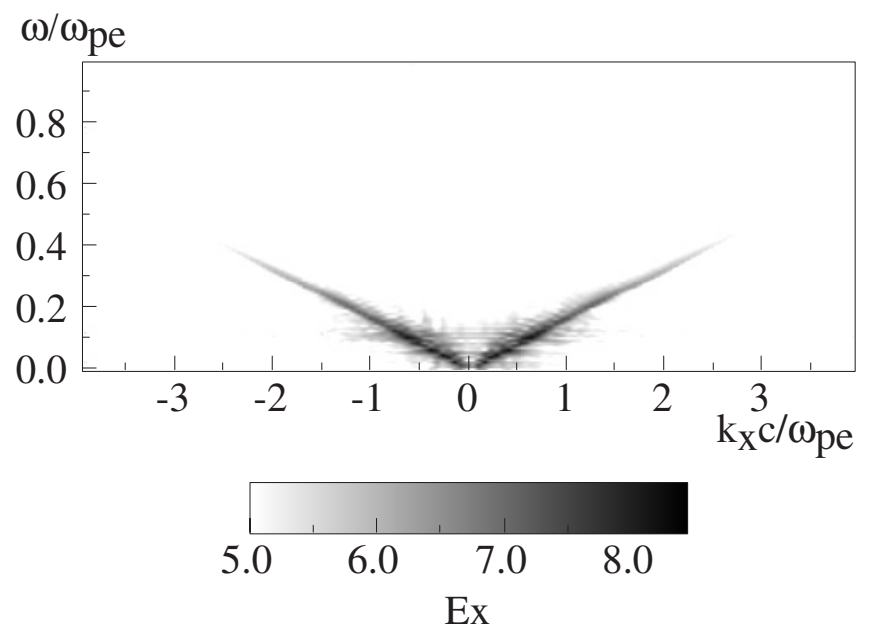

Fig. 4. The dispersion relation of $E_{x}$ obtained by space-time Fourier transformation at $y=400 \Delta$, where the space data is taken in $600 \Delta<$ $x<2648 \Delta$ and the time data is taken in $20 \omega_{\mathrm{ci}}{ }^{-1}<t<45.8 \omega_{\mathrm{ci}}{ }^{-1}$.

is due to the fact that the excited shear AWs propagate with elliptic polarization, while the electron heating in the $x$-direction is due to small-scale electric fields driven by the MTSI.
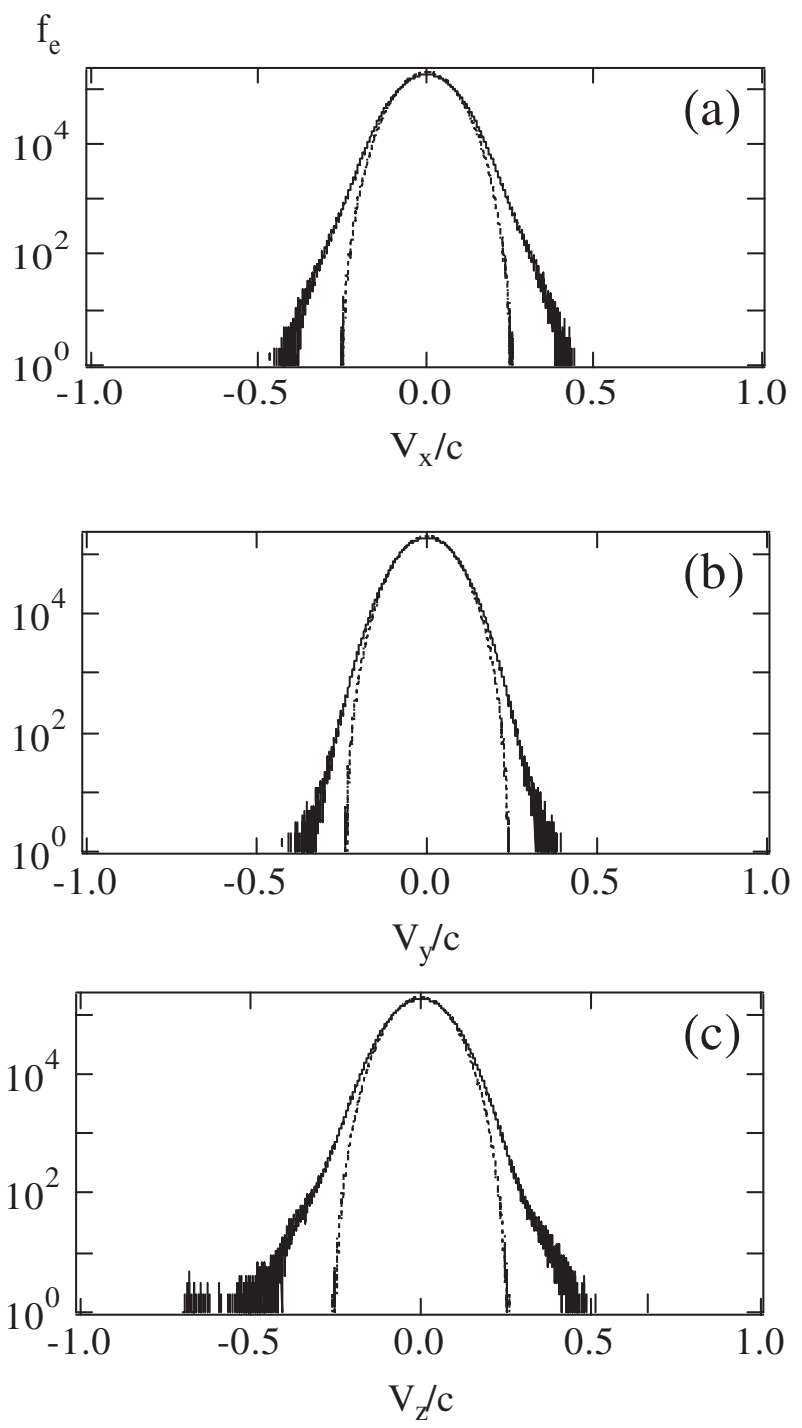

Fig. 5. The electron velocity distribution functions of three components at $\omega_{\mathrm{ci}} t=0$ (dotted line) and 46.87 (solid line).

New observations (Aschwanden et al. 2000) of coronal loops in EUV wavelengths with the TRACE demonstrate that the most influential model of coronal loops originated from Rosner et al. (1978), assuming that a constant pressure along the loop is inapplicable to a class of EUV loops. These loops are found to be heated near the foot-points, with a heating scale height of $12 \pm 5 \mathrm{Mm}$. This supports coronal heating mechanisms operating in or near the chromosphere and transition region. We applied the simulation results obtained in this section to the upper chromosphere. The condition, $v_{\mathrm{A}}>v_{\mathrm{th}, \mathrm{e}}$ is satisfied if the electron temperature is $T_{\mathrm{e}}<6 \times 10^{4} \mathrm{~K}$, where we used the magnetic field $B_{0}=100 \mathrm{G}$ and electron number density $n_{\mathrm{e}}=5 \times 10^{10} \mathrm{~cm}^{-3}$, considering that the chromospheric height from the photosphere is about $2000 \mathrm{~km}$. The proton-neutral hydrogen collision frequency $v_{\mathrm{pn}}$ in this region (Pontieu \& Haerendel 1998) is about $v_{\mathrm{pn}}=100 \mathrm{~s}^{-1}$, that is, slow enough compared with the collisionless damping frequency $v_{\text {damp }}$ of the shear Alfvén wave that is about $v_{\text {damp }}=\omega_{\text {ci }} / 50=2 \times 10^{4} \mathrm{~s}^{-1}$ (for $B_{0}=100 \mathrm{G}$ ) as shown in the previous section. Therefore the collisionless damping of shear Alfvén wave is very important for heating the chromospheric electrons. 


\section{Summary}

We have shown how large-amplitude shear AWs can heat chromospheric plasma (electrons) with a magnetic force-free configuration by using a 2D3V fully relativistic electromagnetic PIC simulation. We found that the shear Alfvén waves with linear polarization and about one period can excite quasielectrostatic waves driven by the MTSI, resulting in electron heating within a short time $t \simeq 50 \mathrm{\omega}_{\mathrm{ci}}{ }^{-1}$. It is not possible to provide exact theoretical estimate for the electron heating time, but the rough estimation is about 200 times of the growing time of the MTSI; $200 \omega_{\mathrm{pi}}{ }^{-1}\left(1+\omega_{\mathrm{pe}}{ }^{2} / \omega_{\mathrm{ce}}{ }^{2}\right)^{1 / 2}$, which give us about $56 \omega_{\mathrm{ci}}{ }^{-1}$. We need further investigation in order to theoretically estimate the electron heating time. The results could be applied to the electron heating in the upper chromosphere where small-scale magnetic flux tubes are interacting with each other to create shear magnetic field structures between them. The force-free magnetic field configuration with magnetic shear used in the present simulation is simple, therefore more realistic magnetic configuration should be considered in the future.
Acknowledgements. We thank the anonymous referee for constructive comments and suggestions that improved our original paper. This work was carried out by the joint research program of the Solar-Terrestrial Environment Laboratory, Nagoya University.

\section{References}

Aschwanden, M. J., Nightingale, R. W., \& Alexander, D. 2000, ApJ, 541, 1059 Buneman, O. 1993, Computer Space Plasma Physics, Simulation Techniques and Software (New York: Terra Scientific), p. 67

De Pontieu, B., \& Haerendel, G. 1998, A\&A, 338, 729

Furusawa, K., \& Sakai, J. I. 2000, ApJ, 540, 1156

Heyvaerts, J., \& Priest, E. 1983, A\&A, 117, 220

Narain, U., \& Ulmschneider, P. 1990, Space Sci. Rev., 54, 377

Narain, U., \& Ulmschneider, P. 1996, Space Sci. Rev., 75, 453

Ott, E., McBride, J. B., Orens, J. H., \& Boris, J. P. 1972, Phys. Rev. Lett., 28, 88

Sakai, J. I., Kawata, T., Yoshida, K., Furusawa, K., \& Cramer, N. F. 2000, ApJ, 537, 1063

Sakai, J. I., Takahata, A., \& Sokolov, I. V. 2001, ApJ, 556, 905

Sakai, J. I., Yamamura, W., Saito, S., et al. 2005, New J. Phys. 7, 233

Tsiklauri, D., Sakai, J. I., \& Saito, S. 2005a, New J. Phys., 7, 79

Tsiklauri, D., Sakai, J. I., \& Saito, S. 2005b, A\&A, 435, 1105 